\title{
A pilot study on faecal MMP-9: a new noninvasive diagnostic marker of colorectal cancer
}

\author{
Anita Annaházi ${ }^{*}, 1,5$, Szabolcs Ábrahám ${ }^{2,5}$, Klaudia Farkas ${ }^{1}$, András Rosztóczy ${ }^{1}$, Orsolya Inczefi ${ }^{1}$, Imre Földesi ${ }^{1}$, \\ Mónika Szücs ${ }^{3}$, Mariann Rutka ${ }^{1}$, Vassilia Theodorou ${ }^{4}$, Helene Eutamene ${ }^{4}$, Lionel Bueno ${ }^{4}$, György Lázár ${ }^{2}$, Tibor \\ Wittmann ${ }^{1}$, Tamás Molnár ${ }^{1}$ and Richárd Róka ${ }^{1}$ \\ ${ }^{1}$ First Department of Medicine, University of Szeged, Korányi Fasor 8-10, Szeged 6720, Hungary; ${ }^{2}$ Department of Surgery, \\ University of Szeged, Pf. 427, Szeged 6701, Hungary; ${ }^{3}$ Department of Medical Physics and Informatics, University of Szeged, \\ Korányi Fasor 9, Szeged 6720, Hungary and ${ }^{4}$ Toxalim UMR 1331 INRA/INP/UPS, Neuro-Gastroenterology and Nutrition Unit, 180, \\ Chemin de Tournefeuille, BP.93173, Toulouse Cedex 3, 31027, France
}

Background: Colorectal cancer (CRC) is one of the leading malignancies worldwide, therefore cheap noninvasive screening methods are of great importance. Matrix-metalloproteinase-9 (MMP-9) has a role in the progression of CRC, and its level is elevated in tumour biopsies. Faecal MMP-9 levels are increased in active ulcerative colitis patients, but in CRC patients, they have never been measured. We aimed to assess the faecal MMP-9 levels in patients undergoing total colonoscopy according to endoscopic and histological diagnosis.

Methods: One hundred and nine patients provided faecal samples for MMP-9 analysis. A total colonoscopy was performed; suspicious lesions were evaluated by histology. Faecal MMP-9 levels were measured by ELISA.

Results: The number of patients allocated to different groups were: negative/diverticulosis: 34 (referred to as controls); hyperplastic polyps: 15; adenomas: 32 (22 at high risk); and CRC: 28. Faecal MMP-9 was significantly increased in CRC compared with all other groups $(P<0.001)$. Faecal MMP-9 was suitable to distinguish CRC patients from controls (sensitivity: 89.3\%; specificity: 91.2\%). By means of a lower cutoff level, faecal MMP-9 identified high-risk adenomas besides CRC (sensitivity: 76\%; specificity: $85.3 \%)$. This lower cutoff level screened $59 \%$ of high-risk adenomas.

Conclusions: Faecal MMP-9 may be a promising new noninvasive marker in CRC.

Colorectal cancer (CRC) represents a major cause of morbidity and mortality in Western societies, and the incidence in developing countries is also rapidly growing (Sung et al, 2008; Siegel et al, 2012). In Europe, CRC is the second most frequent type of malignancy among males and females, with an estimated incidence of $>400000$ new cases per year (Ferlay et al, 2013). The high frequency of the disease and the fact that the prognosis correlates well with the stage at diagnosis makes CRC screening extremely important. Colonoscopy is the gold standard for CRC screening by its high sensitivity and specificity but has also disadvantages, such as risk of complications, high costs and lower acceptance by patients as a screening method. Therefore, noninvasive methods are required to identify patients at high risk of CRC where colonoscopy needs to be performed.

CRC screening recommendations vary between countries but mostly consist of an annual or biannual faecal occult blood test (FOBT) combined with colonoscopy in case of positivity or a colonoscopy every 10 years, in the population aged $>50$ years

\footnotetext{
*Correspondence: Dr A Annaházi; E-mail: annanita3@yahoo.com

${ }^{5}$ These authors contributed equally to this work.
}

Received 23 March 2015; revised 6 January 2016; accepted 20 January 2016; published online 23 February 2016

(c) 2016 Cancer Research UK. All rights reserved 0007 - 0920/16 
(Binefa et al, 2014). Two basic types of FOBT exist, based on the imperceptible intermittent leakage of blood from CRC and highrisk adenomas in stool (Binefa et al, 2014). The guaiac test (gFOBT) detects the peroxidase activity in the haemoglobin haeme subunit, therefore it is nonspecific for human haemoglobin and theoretically requires dietary restrictions a few days before performing the test, but large studies have found no significant advantage of the dietary modifications, therefore lately they are not recommended by many authors (Konrad, 2010). A minimum of two faecal samples are necessary from different stools to obtain an appropriate sensitivity. Sensitivity for CRC ranges from $6.2 \%$ to $83.3 \%$, depending on the test used, while specificity is $>80 \%$ (Binefa et al, 2014). The immunochemical faecal occult blood test (FIT) is based on specific monoclonal or polyclonal antibodies against human haemoglobin, thus it does not require dietary alterations. Contrary to gFOTB, the analysis by FIT can be automated and its threshold value is adjustable by the user. According to the literature, the sensitivity of FIT is extremely variable, from $5.4 \%$ to nearly $98 \%$, depending on the test used (Binefa et al, 2014). Specificity can range from $77 \%$ to $99 \%$. The European Guidelines for quality assurance of CRC recommends the use of FIT rather than gFOBT (Halloran et al, 2012). However, reliable tests with a less variable sensitivity and specificity are still lacking.

Matrix-metalloproteinases (MMPs) are a family of $\mathrm{Zn}^{2+}$. containing endopeptidases, secreted by various cell types, such as tumour cells, mesenchymal cells, fibroblasts and inflammatory cells, such as monocytes, lymphocytes and neutrophils. Increased expression of MMP-9 has been detected in tissue samples from rectal carcinoma and colon cancer, where it correlates with poor prognosis (Unsal et al, 2008; Araujo et al, 2015; Yang et al, 2014). Recently, we have shown that faecal MMP-9 levels are elevated in active ulcerative colitis (UC) patients compared with patients with a functional gastrointestinal disorder (diarrhoea-predominant irritable bowel syndrome) or healthy controls (Annahazi et al, 2013). Faecal MMP-9 levels were found to be a predictor of disease activity in UC patients (Annahazi et al, 2013), and had a high specificity in the diagnosis of pouchitis (Farkas et al, 2014). However, faecal MMP-9 levels in CRC patients have not been investigated.

Based on this background, we aimed to measure faecal MMP-9 levels in patients undergoing colonoscopic examination in our hospital and to analyse the results according to the different colonoscopic and histological findings.

\section{MATERIALS AND METHODS}

Ethical considerations. The study protocol was approved by the Ethical Committee of the University of Szeged, Szeged, Hungary. All subjects provided written and informed consent to participate.

Patient selection and sample collection. Overall, 109 patients (male/female: 58/51, age: 29-91 years, mean: 66.0 years) referred to our specialist colorectal unit in the First Department of Medicine, Szeged, Hungary were enrolled in the study, who all underwent colonoscopy. No patients had a history of UC or a history of other cancers. One patient, who was later identified as a rectal adenocarcinoma patient, had liver metastases. Faecal samples were collected from all patients 2-4 days before the colonoscopy and were frozen to $-20^{\circ} \mathrm{C}$ within $1 \mathrm{~h}$ after defaecation. All colonoscopies were completed until reaching the terminal ileum. During colonoscopy, biopsies were taken from all suspect lesions, and histology was performed. Based on the colonoscopic and histological results, patients were allocated to five groups: negative, diverticulosis, hyperplastic polyp, adenoma, and CRC. Adenomas were considered at increased risk of malignisation when one of the followings was fulfilled: polyp size $>10 \mathrm{~mm}$; villous adenoma or tubulovillous adenoma with at least $20 \%$ villous component; highgrade dysplasia; or multiple adenoma $(>3)$.

Validation assay. Our group was the first to use the commercial Human Quantitative Enzyme-Linked Immunosorbent Assay Kit from R\&D Systems (Abingdon, UK, Cat. No. DMP900) for the measurement of MMP-9 levels in faeces. Therefore, we have performed a validation assay to test whether the faecal matrix itself has an influence on MMP-9 determination and whether the kit from R\&D Systems is able to determine MMP-9 from human faecal extracts.

Active human MMP-9 full-length protein (Abcam LTD, Cambridge, UK; Cat. No.: ab157344; LOT No: GR218764-1) was added to the extraction medium. Control faecal samples were weighed and $1 \mathrm{ml}$ extraction medium with or without added MMP-9 was given to $0.25 \mathrm{mg}$ sample (the sample to extraction medium ratio was $1: 4$ ). The samples were immediately homogenised for $2 \times 30 \mathrm{~s}$ and incubated for $10 \mathrm{~min}$ at room temperature. Subsequently, all homogenates were centrifuged at $1500 \mathrm{~g}$ for $10 \mathrm{~min}$ followed by a second centrifugation of the supernatants from the first centrifugation step at $10000 \mathrm{~g}$ for $10 \mathrm{~min}$. The final supernatants were processed for linearity (parallelism) test. The extract containing added MMP-9 was serially diluted $1+1$ to get $50,25,12.5$ and $6.25 \%$ of the original MMP-9 content with the extract of the same faeces containing no added MMP-9. Then all samples, including $100 \%$ of added MMP-9 extract and the native control extract without added MMP-9, were subjected to MMP-9 measurement by the above-mentioned ELISA kit for human MMP-9. The kit is able to detect the human $92 \mathrm{kDa}$ Pro-MMP-9 and $82 \mathrm{kDa}$ active form of MMP-9 as stated by the manufacturer. The added MMP-9 protein obtained for this evaluation was the $92 \mathrm{kDa}$ Pro-MMP-9.

Measurement of faecal MMP-9. Samples were stored on $-20^{\circ} \mathrm{C}$ for a maximum of 3 months and then thawed at $4{ }^{\circ} \mathrm{C}$ for 1 day. In all, $1.0 \mathrm{~g}$ of each faecal sample was diluted, mixed and homogenised in $4 \mathrm{ml}$ of ice-cold Tris-buffer $(0.15 \mathrm{M} \mathrm{NaCl}+20 \mathrm{~mm}$ Tris- $\mathrm{HCl}$, $\mathrm{pH}: 8.3)$. After centrifugation ( $10 \mathrm{~min}, 4500$ r.p.m., $4{ }^{\circ} \mathrm{C}$ ), pellets were discarded and supernatants were recentrifuged $(10 \mathrm{~min}$, $\left.10000 \mathrm{~g} ; 4^{\circ} \mathrm{C}\right)$. The final supernatants were filtered by $0.8-\mu \mathrm{m}$ pore-sized syringe filters, and the aliquots were stored at $-20^{\circ} \mathrm{C}$ until analysed. MMP-9 was measured by the quantitative enzymelinked immunosorbent assay described above.

Statistical analysis. Continuous data were presented as mean \pm s.d. Kruskal-Wallis test was used to compare faecal MMP-9 levels of the different groups (control, hyperplastic polyp, adenoma and CRC). MMP-9 levels of high- and low-risk adenoma subgroups were analysed by the Mann-Whitney test. The different subgroups according to tumour localisation or stage were compared with the Kruskal-Wallis test. Differences were considered statistically significant when $P<0.05$.

Receiver operating characteristic (ROC) curve analysis was performed to determine the predictive power of the MMP-9 level. Area under ROC curve (AUC), confidence interval for AUC, sensitivity and specificity were calculated. The optimal cutoff values were determined using Youden's index, if the AUC was $>0.8$ (Baker and Kramer, 2007) The cutoff level was determined by the maximum value of Youden's index (sensitivity + specificity -1$)$. Statistical analyses were performed by the $\mathrm{R}$ (version 3.1.2) statistical software (https://www.r-project.org/).

\section{RESULTS}

Validation assay. The concentrations measured in $\mathrm{ng} \mathrm{ml}^{-1}$ are shown in Figure 1. The figure clearly shows a linear concentration 
change corresponding to dilution. It indicates that (i) the faecal matrix itself has no influence on MMP-9 determination; and (ii) the kit from R\&D Systems is able to determine MMP-9 from human faecal extracts.

Faecal MMP-9 levels between groups. Seventeen patients (male/ female: 8/9; mean age: 59.7 years) were found negative on total colonoscopy and 17 patients had no other finding from noninflamed diverticulosis (male/female: 3/14; mean age: 71.8 years). There was no significant difference between these two groups in terms of faecal MMP-9 levels $(P=0.49)$, therefore these 34 patients were used as polyp-free/non-malignant controls (mean age: 65.7 years). Out of the other patients, 15 had hyperplastic polyps (male/female: 9/6; mean age: 62.3 years), 32 had adenomas (male/female: 21/11; mean age: 67.0) and 28 had CRC (all adenocarcinomas; male/female: 17/11; mean age: 67.2 years) according to the endoscopic and histological examinations. There were no statistically significant differences between the four groups (controls, hyperplastic polyps, adenomas, CRC) regarding the age. Faecal MMP-9 levels were significantly different between the four groups (Figure 2, $P<0.0001$ ). Faecal samples from control patients had very low MMP-9 levels (mean: $0.118 \pm 0.057 \mathrm{ng} \mathrm{ml}^{-1}$ ). Patients with hyperplastic polyps also presented with low faecal MMP-9 levels (mean: $0.112 \pm 0.073 \mathrm{ng} \mathrm{ml}^{-1}$ ). Adenoma patients

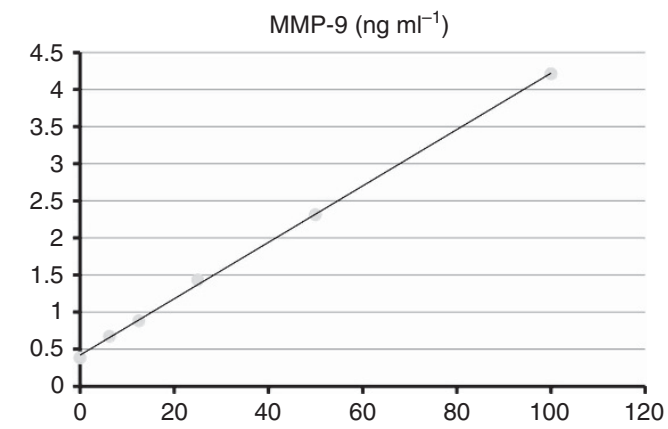

Figure 1. MMP-9 concentration levels at different dilutions in the validation assay.

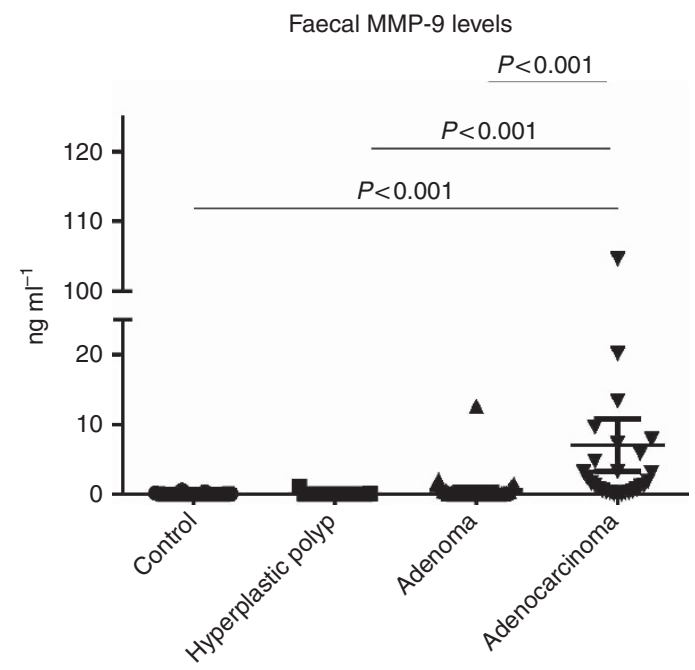

Figure 2. Faecal MMP-9 levels of the different groups (based on colonoscopy and histology). Abbreviation: $\mathrm{CRC}=$ colorectal carcinoma. had slightly, but not significantly elevated faecal MMP-9 levels (mean: $0.656 \pm 0.396 \mathrm{ng} \mathrm{ml}^{-1}$ ). Nevertheless, patients with CRC had a significantly increased mean of faecal MMP-9 levels (mean: $7.028 \pm 3.722 \mathrm{ng} \mathrm{ml}^{-1}$ ). To distinguish CRC patients from controls, the cutoff level was determined at $0.23 \mathrm{ng} \mathrm{ml}^{-1}$ (AUC $=0.913$; 95\% CI: 0.833-0.994; $P<0.001$ ). This cutoff level was associated with a sensitivity of $89.3 \%$ and a specificity of $91.2 \%$ (Figure 3). Patients with hyperplastic polyps (AUC $=0.526 ; 95 \%$ CI: $0.340-0.711 ; P=0.779)$ were not distinguishable from control individuals by ROC analysis. Among adenomas, 22 out of 32 were at increased risk of malignancy, with a mean faecal MMP-9 level significantly elevated compared with low-risk adenomas (mean of high-risk adenomas: $0.936 \pm 0.570$ vs mean of low-risk adenomas: $0.040 \pm 0.017 ; P<0.05$; Figure 4 ). Low-risk adenomas could not be distinguished from controls by faecal MMP-9 levels $(\mathrm{AUC}=0.456$; 95\% CI: $0.236-0.676 ; \quad P=0.706)$ When ROC analysis was performed with an aim to select high-risk adenomas and CRC from controls, the cutoff level was determined at $0.085 \mathrm{ng} \mathrm{ml}^{-1}$. In this case, sensitivity was $76 \%$ and specificity was $85.3 \%$ (AUC $=0.806 ; 95 \%$ CI: $0.710-0.902 ; P<0.001)$. With this lower cutoff level, $59 \%$ of high-risk adenomas were identified. By ROC analysis of high-risk adenomas $v s$ controls, the cutoff level would be similarly $0.085 \mathrm{ng} \mathrm{ml}^{-1}$ (AUC $=0.670 ; 95 \%$ CI: $0.507-0.833$; $P=0.033)$.

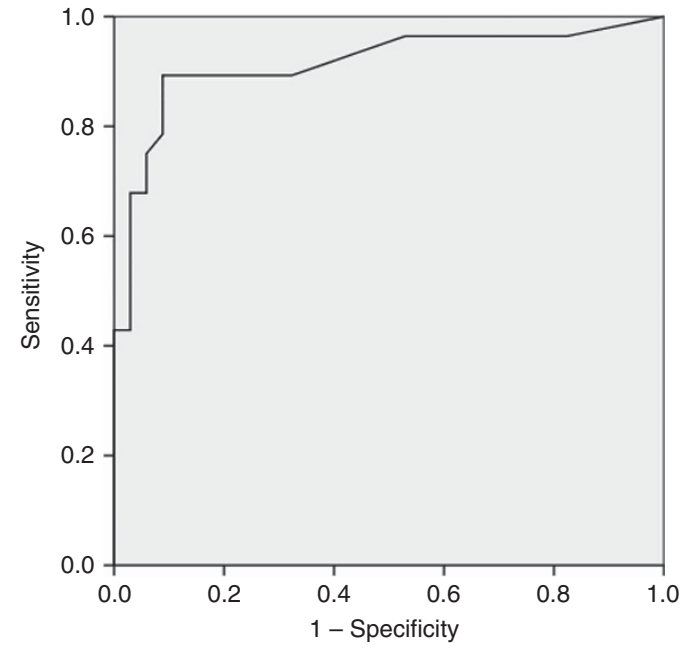

Figure 3. ROC curve of CRC vs controls.

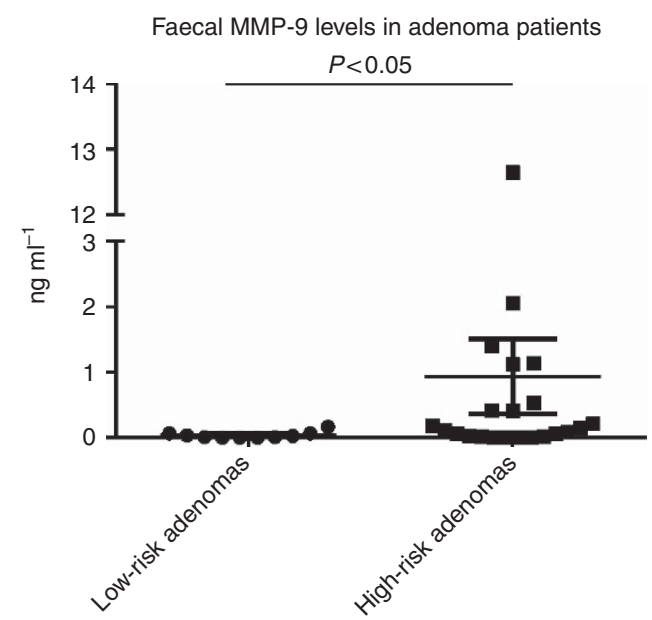

Figure 4. Faecal MMP-9 levels in adenomas. 
A

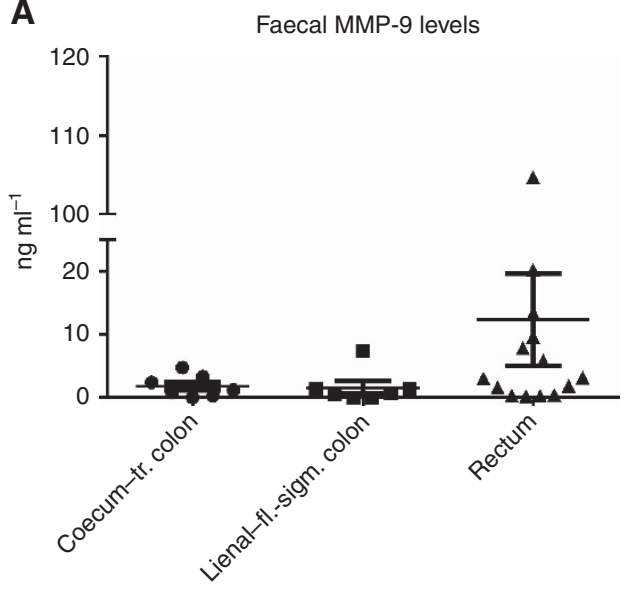

Localisation of adenocarcinoma

B

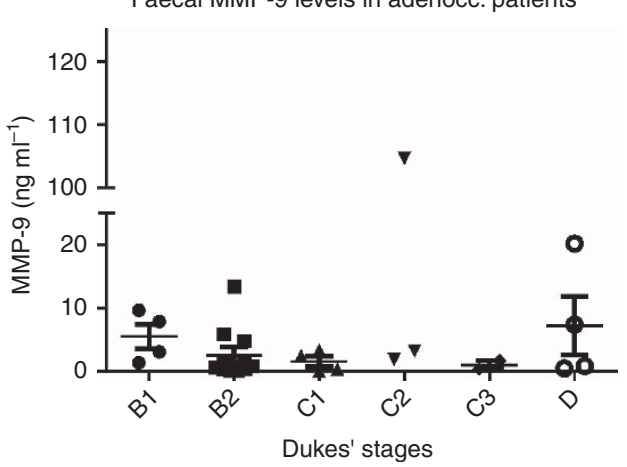

Figure 5. Faecal MMP-9 levels in CRC patients. Values according to (A) localisation and (B) Dukes' stages of CRC.

Faecal MMP-9 levels according to CRC localisation and stage. The number of patients regarding the site of the CRC was as follows: rectum: 14 , sigmoid colon: 6 , lineal flexure: 1 , transverse colon: 2, hepatic flexure: 2 , ascending colon: 1 , and coecum: 2 patients. If faecal MMP-9 levels are analysed according to the site of the CRC, no significant difference was observed $(P=0.146)$, although a trend was seen towards higher levels in case of a rectal tumour (mean: $12.33 \pm 7.274 \mathrm{ng} \mathrm{ml}^{-1}$ ) compared with left-sided colon (lineal flexure-sigmoid colon; mean: $1.619 \pm 0.978 \mathrm{ng} \mathrm{ml}^{-1}$ ) and transverse-right-sided colon (from coecum to transverse colon; mean: $1.830 \pm 0.656 \mathrm{ng} \mathrm{ml}^{-1}$; Figure $5 \mathrm{~A}$ ). According to Dukes' classification, no significant difference could be seen between the groups (Figure 5B; $P=0.236$ ).

\section{DISCUSSION}

To the best of our knowledge, we report for the first time that MMP-9 levels are elevated in faecal specimens from CRC patients compared with patients without polypoid lesions. Furthermore, faecal MMP-9 can be used to distinguish CRC patients from patients with no polypoid lesions, with a high sensitivity and specificity. Additionally, with a lower cutoff level, faecal MMP-9 is able to identify nearly $60 \%$ of patients with a high-risk adenoma.

MMP-9 is a gelatinase capable of the degradation of the extracellular matrix, which is believed to have a role in the progression and metastasis formation of many tumours, including colon cancer (Yang et al, 2014). Apart from colon cancer cells, tumour-infiltrating neutrophils are also an important source of MMP-9, as a major angiogenesis-inducing factor (Hyuga et al,
1994; Deryugina et al, 2014). Serum MMP-9 was suggested as an early noninvasive biomarker for breast cancer (Provatopoulou et al, 2009) and a prognostic marker for lung cancer (Ylisirnio et al, 2000). Urinary MMPs were found elevated not only in bladder, renal and prostate cancers but also in anatomically distant tumours, including breast, brain, and hematological malignancies (Moses et al, 1998; Chan et al, 2004; Fernandez et al, 2005; Smith et al, 2008). Recently, significant efforts have been made to explore the potential role of MMP-9 as a biomarker in CRC. In the serum, it has been suggested as a biomarker of CRC in symptomatic patients (Hurst et al, 2007), and its levels correlated with the Dukes' stage (Mroczko et al, 2010). Furthermore, it has been recently shown that serum levels of neutrophil gelatinaseassociated lipocalin in complex with MMP-9 are elevated in CRC patients, but it was found unsuitable as a diagnostic marker, as its discriminative power was very poor (Duvillard et al, 2014). We have found the discriminative power of faecal MMP-9 levels in CRC patients sufficient, which can be explained by the fact that faecal MMP-9 could be more informative for gastrointestinal malignancies than urine or serum. Our method does not require a preceding diet, and one faecal sample gave a sufficient sensitivity for discrimination. Its analysis is automated, and the cut of concentration is adjustable. In terms of these characteristics, faecal MMP-9 measurement, similarly to FIT, is more convenient for clinical use than gFOBT. Furthermore, faecal MMP-9 measurement provides a higher sensitivity and specificity for CRC than gFOBT and than many types of FIT.

In the past decade, numerous faecal molecular markers have been described, which are based on the detection of genetic mutations and epigenetic alterations, linked to the carcinogenetic process (for a review, see Kanthan et al, 2012). Genetic markers are claimed to be more sensitive than FOBTs (Kanthan et al, 2012). However, one disadvantage of these markers is that they are not present in every colorectal carcinoma. To overcome this problem, genetic markers are often combined in a panel, but this may lead to a decrease in specificity (Kanthan et al, 2012). Furthermore, an important limitation of these methods is their high costs. Current evidence is not sufficient to accept the routine use of faecal genetic markers, and the European Guidelines for quality assurance have stated that they are currently unsuitable for CRC screening (Halloran et al, 2012). Similarly to molecular tests, our method also has the advantage over FOBTs in that it detects a marker originating directly from cancer, which, contrary to bleeding, is supposed to be permanent. However, the costs of faecal MMP-9 measurement are far beyond those of molecular tests, as they are $<10$ USD per sample, which is comparable to the costs of FOBT. This method is markedly cheaper than faecal DNA tests, which cost around 350 USD per sample (Lansdorp-Vogelaar et al, 2010).

The process of transformation from normal epithelium to cancer takes 10-15 years in most cases, leaving a possibility to remove precancerous lesions before they turn to definitive cancer (Binefa et al, 2014). Therefore, a reliable marker detecting high-risk adenomas is of high importance. The sensitivity of FIT for advanced adenomas is only around 20-33\% (Morikawa et al, 2005; Haug et al, 2010). DNA tests are characterised by a much higher sensitivity, for example, K-ras mutations are detected in the faeces of around $50 \%$ of individuals with adenomas $>1 \mathrm{~cm}$ (Mak et al, 2004), p53 mutations in up to $64 \%$ of severely dysplastic polyps (Tagore et al, 2004) and 4-26\% of adenomas (Mak et al, 2004). Methods based on the detection of epigenetic changes appear to have a wide range of sensitivity, for example, the methylation of SFRP2 can be detected in the faeces of $52.4 \%$ of individuals with adenomas (Huang et al, 2007), while the methylation of methylguanine DNA methyltransferase can be found in $36 \%$ of the faeces of adenoma patients but that of the human mut I homolog-1 in only $11 \%$ (Baek et al, 2009). Faecal tumour M2 pyruvate kinase, a promising marker in CRC screening, showed a 
sensitivity of $22 \%$ for advanced adenomas (Haug et al, 2008). Serum MMP-9 levels were elevated not only in cancer but also in colorectal adenoma patients compared with healthy subjects (Mroczko et al, 2010). Similarly, MMP-9 expression was found increased not only in tissue samples from CRC but also in those from highly dysplastic adenomatous polyps compared with hyperplastic polyps or adenomas with low dysplasia (Odabasi et al, 2014). Furthermore, MMP-9 protein expression has been elevated in adenomas with high grade dysplasia compared with other adenomas or normal colonic tissue (Herszenyi et al, 2008). Our results have shown that faecal MMP-9 levels from high-risk adenoma patients are significantly elevated compared with patients with low-risk adenomas. However, with a lower cutoff level, our marker identified 59\% of high-risk adenoma patients as positive. This result is definitely higher than the usual sensitivity of FOBTs and comparable to that of molecular tests but without the high costs of the latter.

We did not find any significant difference between the faecal MMP-9 levels of the groups with different tumour location. Nevertheless, a tendency could be seen towards the highest faecal MMP-9 levels in rectal adenocarcinoma patients. This is probably not related to the nature of rectal tumours but could be explained with a less time for degradation of the marker in the intestinal lumen, similarly to that seen by FIT (Morikawa et al, 2005).

Our study has some limitations. As our department is a specific colorectal center, our patients were a selected population not representing the general population. Patients were on a low-fibre diet at the time of faecal sample collection, as a preparation for the upcoming colonoscopy. Data on the time of the day at faecal sample production was not collected in our study. Although the percentage of cancer and adenoma found on colonoscopy was relatively high, the number of patients in the different subgroups (i.e., tumour localisation, Dukes' stage) may be insufficient for the detection of minor differences. Moreover, MMP-9 is also elevated in gut inflammation, which may limit its use in case of, for example, IBD. We hypothesise that, in a more general population, elevated faecal MMP-9 levels in some cases could be related to inflammation or other cancers in the gastrointestinal tract. However, even in this case, the method would be a good predictor of the presence of a nonfunctional gastrointestinal disorder, based on our previous results showing that faecal MMP-9 measurement can well discriminate IBS-D patients and healthy subjects from UC patients. A further limitation is that, owing to the different cutoff levels calculated on the same sample groups, the performance of the method may be overestimated. A further study with a larger and more general patient population could help us to clarify whether diet, time of the day at sampling, tumours of the upper GI tract and so on can affect faecal MMP-9 levels and could also serve to verify the performance of our method. A comparison with FIT on the same samples could be useful. Moreover, a larger study on a general cohort could also provide more information if this method is valuable for CRC screening.

Nonetheless, our study was suitable to identify a new marker, faecal MMP-9, capable of discrimination of CRC from polyp-free controls. Faecal MMP-9 is characterised by many advantages of FIT and also of the molecular markers, without high costs. Further studies on a larger and more general population are needed to confirm whether this promising method is suitable for CRC screening as a complementary assay.

\section{ACKNOWLEDGEMENTS}

This work was supported by the institutional grants from TÁMOP (TAMOP-4.2.2.A-11/1/KONV-2012-0035, TAMOP-4.2.2-A-11/1/ KONV-2012-0052 TAMOP-4.2.2.A-11/1/KONV-2012-0073).
CONFLICT OF INTEREST

The authors declare no conflict of interest.

\section{REFERENCES}

Annahazi A, Molnar T, Farkas K, Rosztoczy A, Izbeki F, Gecse K, Inczefi O, Nagy F, Foldesi I, Szucs M, Dabek M, Ferrier L, Theodorou V, Bueno L, Wittmann T, Roka R (2013) Fecal MMP-9: a new noninvasive differential diagnostic and activity marker in ulcerative colitis. Inflamm Bowel Dis 19(2): 316-320.

Araujo Jr RF, Lira GA, Vilaca JA, Guedes HG, Leitao MC, Lucena HF, Ramos CC (2015) Prognostic and diagnostic implications of MMP-2, MMP-9, and VEGF-alpha expressions in colorectal cancer. Pathol Res Pract 211(1): 71-77.

Baek YH, Chang E, Kim YJ, Kim BK, Sohn JH, Park DI (2009) Stool methylation-specific polymerase chain reaction assay for the detection of colorectal neoplasia in Korean patients. Dis Colon Rectum 52(8): 1452-1459; discussion 1459-63.

Baker GS, Kramer BS (2007) Peirce, Youden, and receiver operating characteristic curves. Am Stat 61(4): 343-346.

Binefa G, Rodriguez-Moranta F, Teule A, Medina-Hayas M (2014) Colorectal cancer: from prevention to personalized medicine. World $J$ Gastroenterol 20(22): 6786-6808.

Chan LW, Moses MA, Goley E, Sproull M, Muanza T, Coleman CN, Figg WD, Albert PS, Menard C, Camphausen K (2004) Urinary VEGF and MMP levels as predictive markers of 1-year progression-free survival in cancer patients treated with radiation therapy: a longitudinal study of protein kinetics throughout tumor progression and therapy. J Clin Oncol 22(3): 499-506.

Deryugina EI, Zajac E, Juncker-Jensen A, Kupriyanova TA, Welter L, Quigley JP (2014) Tissue-infiltrating neutrophils constitute the major in vivo source of angiogenesis-inducing MMP-9 in the tumor microenvironment. Neoplasia 16(10): 771-788.

Duvillard L, Ortega-Deballon P, Bourredjem A, Scherrer ML, Mantion G, Delhorme JB, Deguelte-Lardiere S, Petit JM, Bonithon-Kopp C (2014) A case-control study of pre-operative levels of serum neutrophil gelatinaseassociated lipocalin and other potential inflammatory markers in colorectal cancer. BMC Cancer 14: 912.

Farkas K, Balint A, Bor R, Foldesi I, Szucs M, Nagy F, Szepes Z, Annahazi A, Roka R, Molnar T (2014) Faecal matrix metalloprotease-9 is a more sensitive marker for diagnosing pouchitis than faecal calprotectin: results from a pilot study. Expert Rev Gastroenterol Hepatol 9(3): 387-392.

Ferlay J, Steliarova-Foucher E, Lortet-Tieulent J, Rosso S, Coebergh JW, Comber H, Forman D, Bray F (2013) Cancer incidence and mortality patterns in Europe: estimates for 40 countries in 2012. Eur J Cancer 49(6): 1374-1403.

Fernandez CA, Yan L, Louis G, Yang J, Kutok JL, Moses MA (2005) The matrix metalloproteinase-9/neutrophil gelatinase-associated lipocalin complex plays a role in breast tumor growth and is present in the urine of breast cancer patients. Clin Cancer Res 11(15): 5390-5395.

Halloran SP, Launoy G, Zappa M (2012) European guidelines for quality assurance in colorectal cancer screening and diagnosis. First EditionFaecal occult blood testing. Endoscopy 44(Suppl 3): SE65-SE87.

Haug U, Hundt S, Brenner H (2008) Sensitivity and specificity of faecal tumour M2 pyruvate kinase for detection of colorectal adenomas in a large screening study. Br J Cancer 99(1): 133-135.

Haug U, Hundt S, Brenner H (2010) Quantitative immunochemical fecal occult blood testing for colorectal adenoma detection: evaluation in the target population of screening and comparison with qualitative tests. Am J Gastroenterol 105(3): 682-690.

Herszenyi L, Sipos F, Galamb O, Solymosi N, Hritz I, Miheller P, Berczi L, Molnar B, Tulassay Z (2008) Matrix metalloproteinase-9 expression in the normal mucosa-adenoma-dysplasia-adenocarcinoma sequence of the colon. Pathol Oncol Res 14(1): 31-37.

Huang Z, Li L, Wang J (2007) Hypermethylation of SFRP2 as a potential marker for stool-based detection of colorectal cancer and precancerous lesions. Dig Dis Sci 52(9): 2287-2291.

Hurst NG, Stocken DD, Wilson S, Keh C, Wakelam MJ, Ismail T (2007) Elevated serum matrix metalloproteinase 9 (MMP-9) concentration 
predicts the presence of colorectal neoplasia in symptomatic patients. $\mathrm{Br} J$ Cancer 97(7): 971-977.

Hyuga S, Nishikawa Y, Sakata K, Tanaka H, Yamagata S, Sugita K, Saga S, Matsuyama M, Shimizu S (1994) Autocrine factor enhancing the secretion of $M(r)$ 95,000 gelatinase (matrix metalloproteinase 9) in serum-free medium conditioned with murine metastatic colon carcinoma cells. Cancer Res 54(13): 3611-3616.

Kanthan R, Senger JL, Kanthan SC (2012) Fecal molecular markers for colorectal cancer screening. Gastroenterol Res Pract 2012: 184343.

Konrad G (2010) Dietary interventions for fecal occult blood test screening: systematic review of the literature. Can Fam Physician 56(3): 229-238.

Lansdorp-Vogelaar I, Kuntz KM, Knudsen AB, Wilschut JA, Zauber AG, van Ballegooijen M (2010) Stool DNA testing to screen for colorectal cancer in the Medicare population: a cost-effectiveness analysis. Ann Intern Med 153(6): 368-377.

Mak T, Lalloo F, Evans DG, Hill J (2004) Molecular stool screening for colorectal cancer. Br J Surg 91(7): 790-800.

Morikawa T, Kato J, Yamaji Y, Wada R, Mitsushima T, Shiratori Y (2005) A comparison of the immunochemical fecal occult blood test and total colonoscopy in the asymptomatic population. Gastroenterology 129(2): $422-428$.

Moses MA, Wiederschain D, Loughlin KR, Zurakowski D, Lamb CC, Freeman MR (1998) Increased incidence of matrix metalloproteinases in urine of cancer patients. Cancer Res 58(7): 1395-1399.

Mroczko B, Groblewska M, Okulczyk B, Kedra B, Szmitkowski M (2010) The diagnostic value of matrix metalloproteinase 9 (MMP-9) and tissue inhibitor of matrix metalloproteinases 1 (TIMP-1) determination in the sera of colorectal adenoma and cancer patients. Int J Colorectal Dis 25(10): 1177-1184.

Odabasi M, Yesil A, Ozkara S, Paker N, Ozkan S, Eris C, Yildiz MK, Abuoglu HH, Gunay E, Tekesin K (2014) Role of human neutrophil gelatinase associated lipocalin (NGAL) and Matrix Metalloproteinase-9 (MMP-9) overexpression in neoplastic colon polyps. Int J Clin Exp Med 7(9): 2804-2811.
Provatopoulou X, Gounaris A, Kalogera E, Zagouri F, Flessas I, Goussetis E, Nonni A, Papassotiriou I, Zografos G (2009) Circulating levels of matrix metalloproteinase-9 (MMP-9), neutrophil gelatinase-associated lipocalin (NGAL) and their complex MMP-9/NGAL in breast cancer disease. BMC Cancer 9: 390.

Siegel R, Naishadham D, Jemal A (2012) Cancer statistics, 2012. CA Cancer J Clin 62(1): 10-29.

Smith ER, Zurakowski D, Saad A, Scott RM, Moses MA (2008) Urinary biomarkers predict brain tumor presence and response to therapy. Clin Cancer Res 14(8): 2378-2386.

Sung JJ, Lau JY, Young GP, Sano Y, Chiu HM, Byeon JS, Yeoh KG, Goh KL, Sollano J, Rerknimitr R, Matsuda T, Wu KC, Ng S, Leung SY, Makharia G, Chong VH, Ho KY, Brooks D, Lieberman DA, Chan FK (2008) Asia Pacific consensus recommendations for colorectal cancer screening. Gut 57(8): 1166-1176.

Tagore KS, Levin TR, Lawson MJ (2004) The evolution to stool DNA testing for colorectal cancer. Aliment Pharmacol Ther 19(12): 1225-1233.

Unsal D, Akyurek N, Uner A, Erpolat OP, Han U, Akmansu M, Mentes BB, Dursun A (2008) Gelatinase B expression as a prognostic factor in patients with stage II/III rectal carcinoma treated by postoperative adjuvant therapy. Am J Clin Oncol 31(1): 55-63.

Yang B, Tang F, Zhang B, Zhao Y, Feng J, Rao Z (2014) Matrix metalloproteinase- 9 overexpression is closely related to poor prognosis in patients with colon cancer. World J Surg Oncol 12: 24.

Ylisirnio S, Hoyhtya M, Turpeenniemi-Hujanen T (2000) Serum matrix metalloproteinases $-2,-9$ and tissue inhibitors of metalloproteinases $-1,-2$ in lung cancer-TIMP-1 as a prognostic marker. Anticancer Res 20(2B): 1311-1316.

This work is published under the standard license to publish agreement. After 12 months the work will become freely available and the license terms will switch to a Creative Commons AttributionNonCommercial-Share Alike 4.0 Unported License. 